\title{
The Cytochemical Localization of Adenyl Cyclase Activity in Rat Sublingual Gland
}

\author{
S. K. KIM AND S. S. HAN \\ Research Service, Veterans Administration Hospital, Department of \\ Anatomy, Medical School, and School of Dentistry, The University \\ of Michigan, Ann Arbor, Michigan 48105
}

\begin{abstract}
Adenyl cyclase activity in mucous acinar cells and serous demilune cells of the rat sublingual gland was localized cytochemically. After incubation with adenylyl-imidodiphosphate (AMP-PNP) as substrate, deposits of reaction product are found along the cell membranes bordering the secretory surfaces of serous demilune cells. These are the membranes which participate directly in secretion by fusing with the granule membranes. The granule membranes of the demilune cells do not reveal reaction product, but the membranes of the granules which are fused with and become part of the cell membrane do show deposits. Thus, it appears that the cell membranes which fuse with granule membranes during secretion are associated with a high level of adenyl cyclase activity. In support of this, the luminal membranes of the mucous acinar cells which do not fuse with granule membranes during secretion are not associated with detectable amounts of adenyl cyclase activity.
\end{abstract}

Adenosine 3',5'-monophosphate (Cyclic AMP) has been established as an intracellular second messenger which mediates the effects of a variety of hormones and other biologically active agents (Jost and Rickenberg, '71; Robison et al., '68; Sutherland et al., '68). Cyclic AMP appears to be also an intermediate in the salivary gland secretion, as demonstrated in the rat parotid gland (Babad et al., '67; Bdolah and Schramm, '65).

Adenyl cyclase catalizes the formation of Cyclic AMP from adenosine triphosphate (ATP) (Sutherland et al., '62). Therefore, it is likely that adenyl cyclase, or factors controlling this enzyme activity, have a significant role in cellular secretory function. In fact, it has been suggested that one major function of adenyl cyclase is the regulation of secretion (Rasmussen and Tenenhouse, '68). Furthermore, adenyl cyclase appears to be localized in cell membranes (Davoren and Sutherland, '63) which participate directly in the granule discharge from serous secretory cells. In these cells, the granule discharge occurs by the fusion of the acinar cell membrane of the secretory surfaces with the granule membrane
(Amsterdam et al,, '69; Hand, '70; Ichikawa, '65).

Previous biochemical studies of the rat parotid gland have indicated that adenyl cyclase is associated largely with cell membranes (Schramm and Naim, '70). However, it is unknown whether the enzyme activity is associated with the entire cell membrane or with certain portions of the membrane, i.e., the apical cell membrane which fuses with the granule membrane during secretion. Also, it is unknown whether or not the enzyme activity is affected as the cell membrane becomes modified by the addition of granule membranes during the secretory process.

Recent studies have shown that adenyl cyclase activity can be demonstrated cytochemically at the electron microscope level in the liver (Reik et al., '70), and in the islets of Langerhans of the pancreas (Howell and Whitfield, '72). Furthermore, it has been shown that the specificity of the cytochemical method can be increased by using an artificial substrate, adenylylimidodiphosphate (AMP-PNP), instead of ATP (Howell and Whitfield, '72). AMP-

Accepted June 16, '75. 
PNP, an analogue of ATP in which nitrogen is substituted for oxygen between the terminal phosphates, serves effectively as substrate for adenyl cyclase but not for ATPase (Rodbell et al., '71).

The present study is an attempt to localize cytochemically adenyl cyclase activity in salivary gland cells. The rat sublingual gland was used for this study so that the localization of adenyl cyclase activity can be compared in mucous acinar cells and serous demilune cells. Such a comparison would be interesting in view of the apparent differences in the secretory mechanism of these two types of cells. Previous studies have shown that mucous granules are discharged through gaps formed in the apical cell membrane without involving the fusion of the granule membrane with the cell membrane (Hollman, '63; Freeman, '66; Neutra and Leblond, '66; Tandler et al., '69; Kim et al., '72). This study indicates that a high level of adenyl cyclase activity is associated with portions of the serous demilune cell membranes which are involved in the discharge processes of the secretory granules but not with similar membranes of the mucous acinar cells. Preliminary results of this study have been published in abstract form (Kim, '74).

\section{MATERIALS AND METHODS}

The procedures used for tissue preparation and incubation in this study are similar to those described by Reik et al. ('70) and Howell and Whitfield ('72). Male Sprague Dawley rats weighing about 200 grams were perfused through the heart with $1 \%$ glutaraldehyde in $0.05 \mathrm{M}$ cacodylate buffer containing $4.5 \%$ glucose $(\mathrm{pH}$ 7.4). Sublingual glands were removed, sliced into small pieces and further fixed in the same fixative. Total fixation time, including perfusion time, was 30-45 minutes at room temperature. Fixed tissues were washed several times in $0.05 \mathrm{M}$ cacodylate buffer containing $8 \%$ glucose and stored in buffer overnight in the refrigerator. The tissues were further sliced into smaller pieces under the dissecting microscope by using a sharp scalpel blade.

The incubation medium consisted of $80 \mathrm{mM}$ Tris-maleate buffer, $\mathrm{pH} 7.4,2 \mathrm{mM}$ theophylline, $2 \mathrm{mM}$ magnesium sulfate and $4 \mathrm{mM}$ lead nitrate. Sodium fluoride (10 $\mathrm{mM}$ ) was added as the activator. In most incubations, $0.5 \mathrm{mM}$ AMP-PNP (obtained from ICN,, Ltd., Irvine, California) was used as substrate. ATP was used as substrate in some incubations for comparison. The tissues were incubated for 35-45 minutes at $30^{\circ} \mathrm{C}$ in a shaker water bath. At the end of the incubation, tissues were rinsed briefly in Tris-maleate buffer and then in cacodylate buffer before fixing with $1 \% \mathrm{OsO}_{4}$ in $0.05 \mathrm{M}$ cacodylate buffer for one hour.

Two types of control incubations were carried out: in one, the tissues were incubated in complete medium without the substrate. In the second control, the incubation medium contained the substrate without sodium fluoride.

After incubation, the tissues were processed for electron microscopy. Sections were cut on a Porter-Blum MT $2 \mathrm{~B}$ ultramicrotome and studied with a Hitachi $11 \mathrm{c}$ electron microscope after staining either with uranyl acetate and lead citrate or uranyl acetate alone.

\section{RESULTS}

The detailed descriptions of the structure of the rat sublingual gland have appeared previously (Enomoto and Scott, '71; Kim et al., '72). Briefly, the rat sublingual gland is of compound tubulo-acinar structure and consists of secretory acini and ducts. Two types of secretory cells occur in each acinus (fig. 1), mucous acinar cells and serous demilune cells. In each acinus, mucous acinar cells predominate and line the lumen, forming secretory tubules. The demilune cells which are found at the blind end of the tubules appear as crescents in sections and line the terminal portions of the secretory lumen. The demilune cells are similar in appearance to the acinar cells of serous glands such as the parotid and pancreas. The secretory granules of the demilune cells are morphologically similar to zymogen granules.

The lumen of the acinus branches at the terminal portion and traverses between demilune cells where it forms secretory canaliculi. Therefore, the discharge of granules occurs at secretory surfaces bordering the canalicular lumen and its branches. As shown in figure 2, the lumen 
and its secretory canaliculi are both bound by junctional complexes and often lined by microvilli-like projections. Although secretory granules of the demilune cells are found frequently in the vicinity of the lumen and canaliculi, those with fused membranes at the secretory surfaces, as shown in figure 5, are only rarely seen in non-stimulated glands.

Observations of incubated tissues indicate that adenyl cyclase activity occurs associated with secretory surfaces of the demilune cells but not with those of the mucous cells. The reaction product is localized in the extracellular surface along the cell membrane of the demilune cells (figs. 3,4 ). Deposits occur along the luminal surface of the demilune cells, but not along that of the adjacent acinar cells (fig. 3). Heavy deposits also occur along the membranes bordering the secretory canaliculi between two demilune cells (figs. 3, 4).

The enzyme activity has not been detected intracellularly. Neither mucous granules of the acinar cells nor zymogenlike granules of the demilune cells reveal the reaction product. However, it appears that the granule membranes of the demilune cells become positive for adenyl cyclase activity following fusion with the cell membrane. As can be seen in figure 5 , the reaction product is localized along the granule membranes which are fused with the cell membrane at the lumen. The membranes which seem to invaginate into the demilune cells shown in figures 3 and 4 may also be the granule membranes fused with cell membranes.

In the glandular tissue incubated with ATP as substrate, deposits of reaction product are found along the lateral membranes of some secretory cells in addition to the membranes of the secretory surfaces. The control tissues incubated without the substrate do not reveal any reaction product. In the control tissues incubated with the substrate, but without sodium fluoride, the reaction product is found in the same areas as in the tissues incubated with sodium fluoride; however, the amount of reaction product present in tissues incubated without the extra activator was somewhat less than that generated in the presence of the added activator.

\section{DISCUSSION}

Rat sublingual gland tissues incubated for the demonstration of adenyl cyclase activity show reaction product localized along the outer surfaces of the serous demilune cell membranes which border lumina and secretory canaliculi. The reaction product, however, has not been detected in association with the luminal surface of the mucous acinar cell membranes. The lack of reaction product cannot be interpreted necessarily as the absence of enzyme activity; however, the occurrence of the reaction product generated by the enzymes which survive the fixation and cytochemical procedures employed clearly indicates the presence of enzymatic activity. Thus, it appears that a high level of adenyl cyclase activity is associated with the demilune cell membranes which border the lumen and the secretory canaliculi. This seems significant since both areas make up the secretory surfaces.

Various results obtained from this and previous studies indicate that the cytochemical method used for the localization of adenyl cyclase is specific for the enzyme activity. 1. Parallel biochemical and cytochemical studies have indicated that 10$50 \%$ of adenyl cyclase activity survives fixation procedures similar to that employed in this study (Howell and Whitfield, '72; Reik et al., '70). 2. There is a correlation between the amount of precipitate observed in the electron microscope and the enzyme activity determined biochemically (Reik et al., '70). 3. AMP-PNP has been shown to be effective as a substrate for adenyl cyclase but is not cleaved by ATPase or other phosphohydrolases (Rodbell et al., '71). 4. When incubated with ATP as substrate, the reaction product occurs along the lateral cell membranes in addition to the secretory surfaces. 5. The tissues incubated in the medium without the substrate do not reveal any deposit. 6. The deposit present in the gland without the added activator may be interpreted as adenyl cyclase activity generated by the action of endogenous activators.

The presence and absence of detectable enzymatic activity in membranes of the demilune cells and acinar cells, respectively, can be related to the differences in 
the role the membranes of these cells play in secretion processes. It has been demonstrated that the discharge mechanisms of secretory substances in mucous acinar cells and serous demilune cells of the rat sublingual gland differ and that the essential difference lies in the behaviour of their membranes (Kim et al., '72). The mucous granules of the acinar cells discharge through gaps in the apical cell surface as in other mucous cells, such as the goblet cells of the intestine (Freeman, '66; Hollman, '63; Neutra and Leblond, '66), and the acinar cells of the labial gland (Tandler et al., '69). Thus, the acinar cell membrane bordering the lumen does not seem to be involved directly in the secretion process of mucus.

On the other hand, the granules of the demilune cells discharge in the same way as the secretory granules of other serous cells (Amsterdam et al., '69; Hand, '70; Ichikawa, '65). Granule discharge occurs by establishing continuity between the granule membrane and the cell membrane bordering the secretory surfaces. Thus, the presence of a high level of adenyl cyclase activity in the membranes bordering the secretory surfaces implies that the enzyme might be involved in the process of secretion.

It has been suggested previously that adenyl cyclase, or the factors controlling this enzyme activity play a significant role in the regulation of secretion (Schramm and Naim, '70). Obviously, the exact mechanism by which adenyl cyclase is involved in the regulation of secretory processes remains unclear. However, previous studies have indicated that cyclic AMP is an intermediate in the salivary gland secretion, as the secretion of amylase in the parotid gland appears to be mediated by cyclic AMP (Babad et al., '67; Bdolah and Schramm, '65).

The occurrence of reaction product for adenyl cyclase activity along the outer surface of the demilune cell membranes observed in this study suggests that cyclic AMP is released into the cytoplasm of these cells. The cyclic AMP released there could function in facilitating the process of fusion between the granule membrane and the cell membrane by modifying either or both of these membranes. In fact, it has been suggested that cyclic AMP modifies cellular membranes by altering the permeability of these membranes to calcium ions (Rasmussen and Tenenhouse, '68).

Apparently, membrane modifications do occur during secretion in serous secretory cells, at least with respect to adenyl cyclase activity. Schramm and Naim ('70) have shown by biochemical methods that there is no significant amount of adenyl cyclase activity associated with the granule membranes of the rat parotid gland. Our findings support this and suggest that the increased activity associated with the membranes after fusion may represent the membrane modifications.

The apparent high level of adenyl cyclase activity associated with the demilune cell membranes bordering the secretory surfaces might be related to the ability of these membranes to fuse with the granule membranes. Amsterdam et al. ('69) have shown that, in rat parotid glands, a granule membrane fuses with additional granules only after it fuses with and becomes part of the luminal membrane. In addition, the luminal membranes of mucous acinar cells of the rat sublingual gland, for example, do not fuse with the membranes of mucous granules (Kim et al., '72) and no detectable adenyl cyclase activity is observed in association with these membranes.

\section{ACKNOWLEDGMENTS}

The authors appreciate the technical assistance provided by Virginia Hartog as well as her effort in the preparation of this manuscript. Veterans Administration Research Project 4927:01.

\section{LITERATURE CITED}

Amsterdam, A., I. Ohad and M. Schramm 1969 Dynamic changes in the ultrastructure of the acinar cells of the rat parotid gland during the secretory cycle. J. Cell Biol., 41: 753-773.

Babad, H., R. Ben-Zvi, A. Bdolah and M. Schramm 1967 The mechanism of enzyme secretion by the cell. 4. Effects of inducers, substrates and inhibitors on amylase secretion by rat parotid slices. Europ. J. Biochem., 1; 96-101.

Bdolah, A., and M. Schramm 1965 The function of $3^{\prime} 5^{\prime}$ cyclic AMP in enzyme secretion. Biochem. Biophys. Res. Comm., 18: 452-454.

Davoren, P. R., and E. W. Sutherland 1963 The cellular location of adenyl cyclase in the pigeon erythrocyte. J. Biol. Chem., 238: 3016-3023. 
Enomoto, S., and B. L. Scott 1971 Intracellular distribution of mucosubstances in the major sublingual gland of the rat. Anat. Rec., 169: 71-96.

Freeman, J. A, 1966 Goblet cell fine structure. Anat. Rec., 154: 121-148.

Hand, A. W. 1970 The fine structure of von Ebner's gland of the rat. J. Cell Biol., 44: 340353.

Hollman, K. H. 1963 The fine structure of the goblet cells in the rat intestine. Ann. N.Y. Acad. Sci., 106: 545-554.

Howell, S. L., and M. Whitfield 1972 Cytochemical localization of adenyl cyclase activity in rat islets of Langerhans. J. Histochem. Cytochem., 20: 873-879.

Ichikawa, A. 1965 Fine structural changes in response to hormonal stimulation of the perfused canine pancreas. J. Cell Biol., 24: 369385.

Jost, J-P., and H. V. Rickenberg 1974 Cyclic AMP. Ann. Rev. Biochem., 40: 741-774.

Kim, S. K. 1974 The localization of adenyl cyclase in rat sublingual gland. Anat. Rec,, 178: 391.

Kim, S. K., C. E. Nasjleti and S. S. Han 1972 The secretion processes in mucous and serous secretory cells of the rat sublingual gland. J. Ultrastruct. Res., 38: 371-389.

Neutra, M., and C. P. Leblond 1966 Synthesis of the carbohydrates of mucus in the Golgi complex shown by electron microscope radioautography of goblet cells from rats injected with glucose-H ${ }^{3}$. J. Cell Biol., 30: 119-136.

Rasmussen, H., and A. Tenenhouse 1968 Cyclic adenosine monophosphate, $\mathrm{Ca}^{++}$, and membranes. Proc. Natl. Acad. Sci., 59: 1364-1370.

Reik, L., G. L. Petzold, J. A. Higgins, P. Greengard and R. J. Barrnett 1970 Hormone-sensitive adenyl cyclase: Cytochemical localization in rat liver. Science, 168: 382-384.

Robison, G. A., R. W. Butcher and E. W. Sutherland 1968 Cyclic AMP. Ann. Rev. Biochem., 37: 149-175.

Rodbell, M., L. Birnbaumer, S. L. Pohl and H. M. J. Krans 1971 The glucagon-sensitive adenyl cyclase system in plasma membranes of rat liver. J. Biol. Chem., 246: 1877-1882.

Schramm, M., and E. Naim 1970 Adenyl cyclase of rat parotid gland. J. Biol. Chem., 245: 3225-3231.

Sutherland, E. W., T. W. Rall and T. Menon 1962 Adenyl cyclase: 1. Distribution, preparation and properties. J. Biol. Chem., 237: 12201227.

Sutherland, E. W., G. A. Robison and R. W. Butcher 1968 Some aspects of the biological role of adenosine $3^{\prime}, 5^{\prime}$-monophosphate (cyclic AMP ). Circulation, 37: 279-306.

Tandler, B., C. R. Denning, I. D. Mandel and A. H. Kutscher 1969 Ultrastructure of human labial salivary glands. 1. Acinar secretory cells. J. Morph., 127: 383-408.

Note added in proof: Recently an article appeared which showed lead inhibition of adenylate cyclase activity, and claimed that the precipitates observed in lead cytochemistry of the enzyme activity are the artifacts resulting from a nonenzymatic hydrolysis of the substrate ("Pitfalls in the use of lead nitrate for histochemical demonstration of adenylate cyclase activity," A. Lemay and L. Jarett 1975 J. Cell Biol., 65: 39-50). These authors' conclusions, however, are based on the biochemical study of fresh, isolated fat pad capillaries and pancreatic islet homogenates. In the fixed salivary gland examined in the present study, the reaction product occurs consistently in association with only one type of secretory cells and this restricted localization of deposits can not be explained by the nonenzymatic hydrolysis hypothesis. Therefore, the effect of lead on adenylate cyclase activity in fixed and unfixed cells from different tissues requires further examination. 
PLATE 1

EXPLANATION OF FIGURES

Unless stated otherwise, sections are stained with uranyl acetate followed by lead citrate and all calibrations lines represent $1 \mu \mathrm{m}$ in all figures, including those of plate 2 .

1 The terminal portion of an acinus showing the acinar cells ( $A C$ ) and demilune cells (DC). The demilune cells cap the end portions of the secretory tubules which are made up of mucous acinar cells around the Iumen (L). The demilune cells are similar to acinar cells of serous glands in containing dense, zymogen-like granules and large amounts of endoplasmic reticulum. $\times 5,000$.

2 A portion of an acinus showing the lumen (L) and secretory canaliculi (C). Both are bound by junctional complexes, lined by microvilli-like projections and reveal some amorphous materials. A secretory granule (arrow) in contact with the membrane of a canaliculus and may be in the process of discharge. $\times 20,000$. 

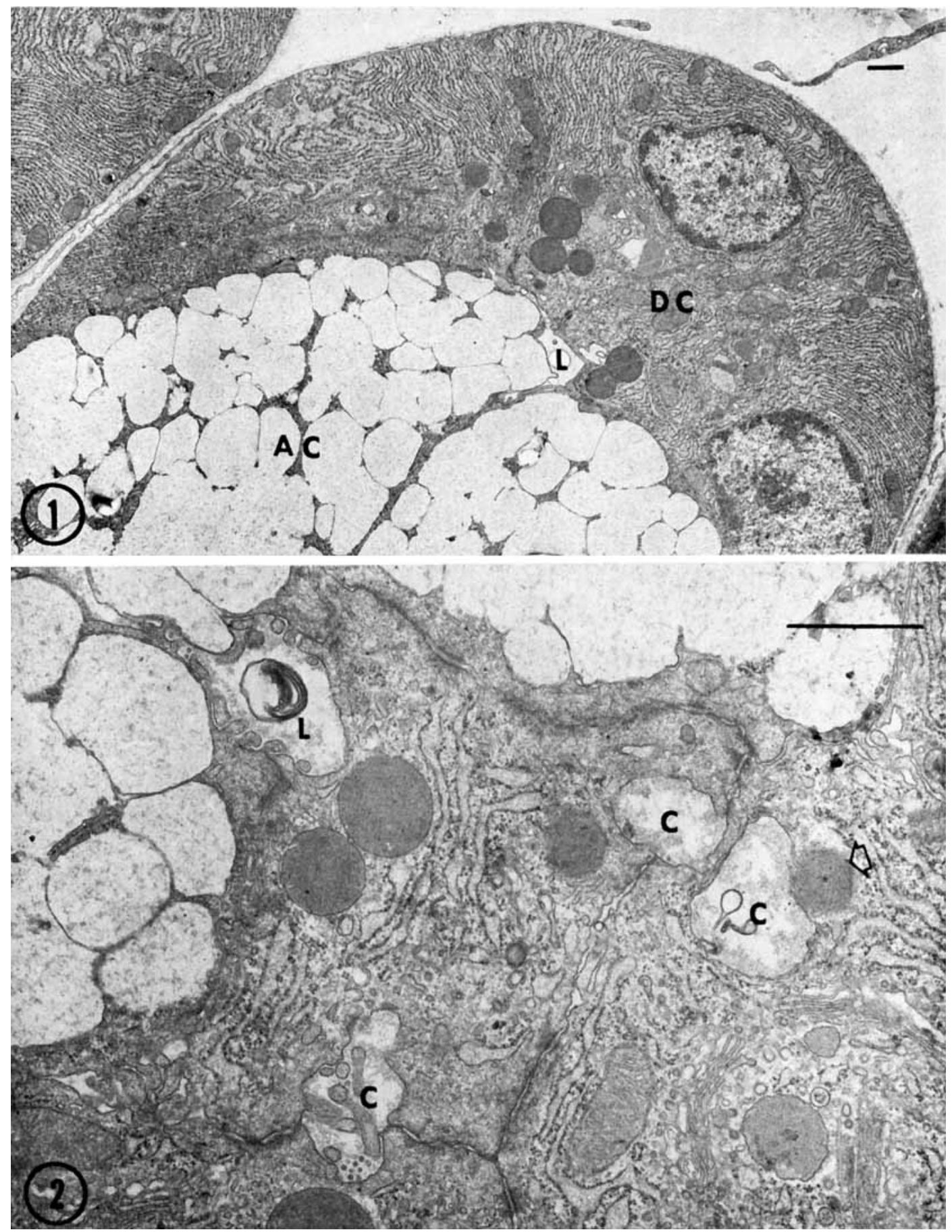
PLATE 2

\section{EXPLANATION OF FIGURES}

Micrographs of tissues incubated for the demonstration of adenyl cyclase activity with AMP-PNP as substrate showing the localization of the reaction product. Sodium fluoride was added as the activator.

3 Deposits of reaction product are localized along the surface of the demilune cell membranes bordering the lumen (DL) and a secretory canaliculus (C). Deposits are absent along the mucous acinar cell membranes which border the lumen (AL). Small vesicle-like structures with reaction product are probably the invaginations of the demilune cell membrane at the lumen. IC indicates a widened area of the intercellular space. $\times 20,000$.

4 The reaction product occurs along the surface of the cell membrane bordering the secretory canaliculi between two demilune cells. Deposits are also present on the surface of the microvilli-like projections. No reaction product is seen in the intercellular space. $\times 20,500$.

5 The luminal surface of a demilune cell is fused with a secretory granule (GA), which in turn is fused with granule (GB) in several sections away. The reaction product occurs along the fused granule membrane which is continuous with the cell membrane. However, the reaction product is absent in the granule (GU) which is not fused with the cell membrane. $L$ indicates the lumen. Stained with uranyl acetate only. Scale $=0.5 \mu \mathrm{m} . \times 45,000$. 

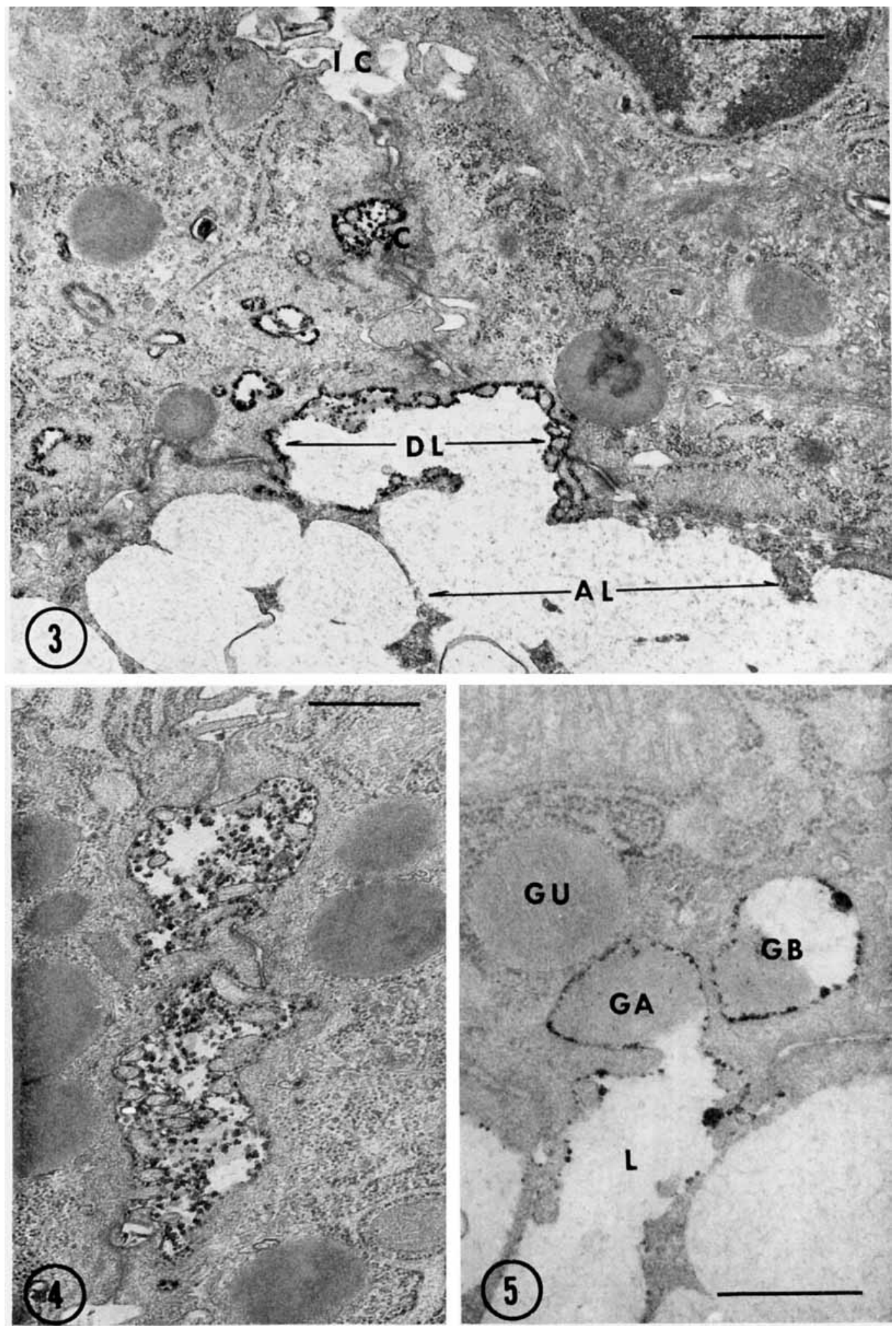